\title{
Opening and Closing of Particle Shells on Droplets via Electric Fields and its Applications
}

Zbigniew Rozynek, ${ }^{\dagger *}$ Khobaib Khobaib, $§$ and Alexander Mikkelsen ${ }^{\S}$

${ }^{\dagger}$ Harvard John A. Paulson School of Engineering and Applied Sciences, Harvard University, Cambridge, MA 02138, USA

$\S$ Faculty of Physics, Adam Mickiewicz University, Umultowska 85, Poznań 61-614, Poland

${ }^{*}$ Corresponding author: zbiroz@seas.harvard.edu 
Table S1. Particle concentration in silicone oil.

\begin{tabular}{|c|c|c|c|}
\hline Figure & Particles & Concentration & Droplet size \\
\hline Fio 2 & green PE (20 um) & $\sim 3 w / w \%$ & $\sim 3.0 \mathrm{~mm}$ \\
\hline Fig. 2 & & $\sim 3 w / w \%$ & $\sim 3.2 \mathrm{~mm}$ \\
\hline Fig. $4 d$ & STC & $\sim 10 \mathrm{w} / \mathrm{w} \%$ & $\sim 3.5 \mathrm{~mm}$ \\
\hline Fig. 4b & green Pt (90 um) & 〜 10w/w\% & $\sim 35 \mathrm{~mm}$ \\
\hline Fig. 5a & green PE (90 um) & $10 \mathrm{~W} / \mathrm{W} \%$ & 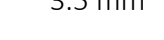 \\
\hline Fig. $5 b$ & silica (250 nm) & $\sim 35 \mathrm{w} / \mathrm{w} \%^{*}$ & $\sim 0.2 \mathrm{~mm}$ \\
\hline Fig. $5 c$ & Li-fluorohectorite ( $\sim \mu \mathrm{m})$ & $\sim 30 \mathrm{w} / \mathrm{w} \%{ }^{*}$ & $\sim 0.5 \mathrm{~mm}$ \\
\hline Fig. 5d & red $P E$ (50 um) & $\sim 7 \mathrm{w} / \mathrm{w} \%$ & $\sim 3.0 \mathrm{~mm}$ \\
\hline Fig. $5 e$ & paramagnetic (50 um) & $\sim 12 \mathrm{w} / \mathrm{w} \%$ & $\sim 2.0 \mathrm{~mm}$ \\
\hline Fig. 6 & red PE (50 um) & $\sim 7 \mathrm{w} / \mathrm{w} \%$ & $\sim 2.0 \mathrm{~mm}$ \\
\hline Fig. 7a & green PE (100 um) & 10 w/w\% & $\sim 2.0 \mathrm{~mm}$ \\
\hline Fig. 7b & red $P E$ (50 um) & $\sim 7 \mathrm{w} / \mathrm{w} \%$ & $\sim 2.0 \mathrm{~mm}$ \\
\hline Fig. 7c & red $P E$ (50 um) & $\sim 7 \mathrm{w} / \mathrm{w} \%$ & $\sim 2.0 \mathrm{~mm}$ \\
\hline Fig. 8a & Li-fluorohectorite ( $\sim \mu \mathrm{m})$ & $\sim 25 \mathrm{w} / \mathrm{w} \%^{*}$ & $\sim 3.0 \mathrm{~mm}$ \\
\hline Fig. 8b & green PE (20 um) & $\sim 3 w / w \%$ & $\sim 3.0 \mathrm{~mm}$ \\
\hline Fig. 8c & red PE (50 um) & $\sim 9 w / w \%$ & $\sim 3.0 \mathrm{~mm}$ \\
\hline Fig. S1 & red PE (50 um) & $\sim 7 \mathrm{w} / \mathrm{w} \%$ & $\sim 1.8 \mathrm{~mm}$ \\
\hline Fig. S2 & red PE (50 um) & $\sim 11 \mathrm{w} / \mathrm{w} \%$ & $\sim 3.0 \mathrm{~mm}$ \\
\hline Fig. S4 & green PE (100 um) & $\sim 10 \mathrm{w} / \mathrm{w} \%$ & $\sim 2.0 \mathrm{~mm}$ \\
\hline Fig. S5 & red $P E$ (50 um) & $\sim 7 \mathrm{w} / \mathrm{w} \%$ & $\sim 3.0 \mathrm{~m}$ \\
\hline Fig. S6 & Laponite ( $5 \mu \mathrm{m})$ & $\sim 25 \mathrm{w} / \mathrm{w} \% *$ & $\sim 5.0 \mathrm{~mm}$ \\
\hline
\end{tabular}

*High concentration was used to prepare the dispersion that was centrifuged to remove particle agglomerates $>10 \mu \mathrm{m}$

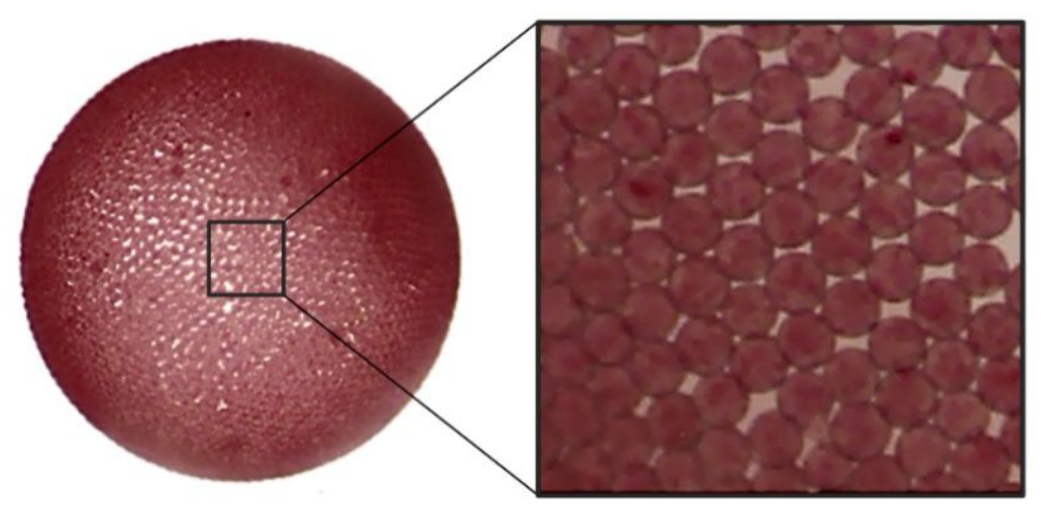

Figure S1. Particle-covered droplets were formed using a DC electric field. An example of a silicone oil droplet covered with PE particles ( $50 \mu \mathrm{m})$. The particles (strongly capillary bound the droplet's surface) were arranged in a jammed, slightly disordered hexagonal structure as shown in the blow-up (see also Movie S1). 

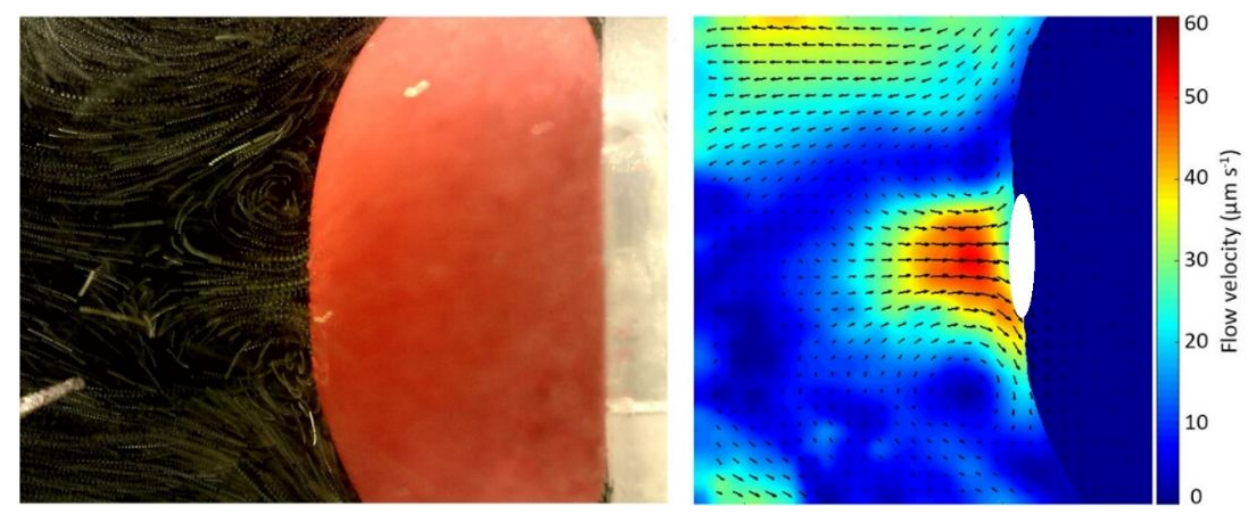

Figure S2. (left) Experimentally observed hydrodynamic streamlines outside a particle-laden droplet while subjected to an electric field of strength $260 \mathrm{~V} \cdot \mathrm{mm}^{-1}$. PE particles $(\sim 20 \mu \mathrm{m})$ were used as tracer particles in the surrounding castor. The sample cell was filled with castor oil and the droplet $(\sim 3 \mathrm{~mm})$ covered with PE particles $(\sim 50 \mu \mathrm{m})$ was attached to an O-ring (fastened at one electrode). The image was composed by overlapping several images captured over a period of 20 seconds. (right) The corresponding particle image velocimetry image shows the flow velocity map. The white area represents the size of the opening in the particle layer.

By performing particle image velocimetry (PIV) experiments, we measured the magnitude and direction of EHD flows quantitatively outside the droplet's electric pole. PE particles ( $20 \mu \mathrm{m})$ were suspended in castor oil and used as tracer particles (see the left panel of Figure S2). When we applied a DC electric field, the particles followed the electric field-induced flows outside the droplet's electric pole. The flow directions and velocities for a droplet subjected to an electric field of strength $260 \mathrm{~V} \cdot \mathrm{mm}^{-1}$ are visualized in the right panel of Figure S2. The flow fields demonstrate how the surface particles on the droplet are carried away from the droplet's electric pole by the flow. Two vortexes are located close to the droplet interface, and the flow velocity is fastest closer to the droplet's electric pole (magnitude around 50 $\left.\mu \mathrm{m} \cdot \mathrm{s}^{-1}\right)$. The flows outside the droplet were measured after the droplet had reached a steady-state deformation and no further structuring of the particle layer was observed.

(a)

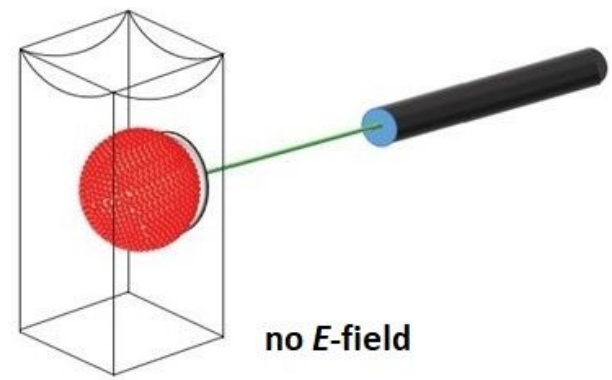

(b)

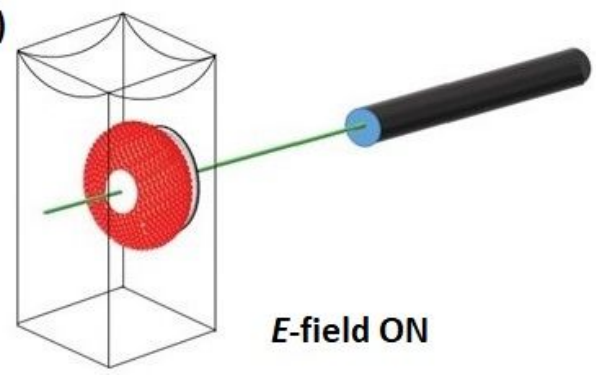

Figure S3. (a,b) Experimental setup for a laser experiment. A sample cell $(10 \mathrm{~mm} \times 10 \mathrm{~mm} \times 30 \mathrm{~mm})$ was made of glass with two of the inside walls coated with a conductive indium tin oxide (ITO) layer, constituting electrodes. An O-ring of size $4 \mathrm{~mm}$ was fastened on the inside of the sample cell to one of the electrodes. The sample cell was filled with castor oil, and a silicone oil droplet covered with PE particles was docked in the O-ring. The droplet was illuminated with laser light (class III laser 


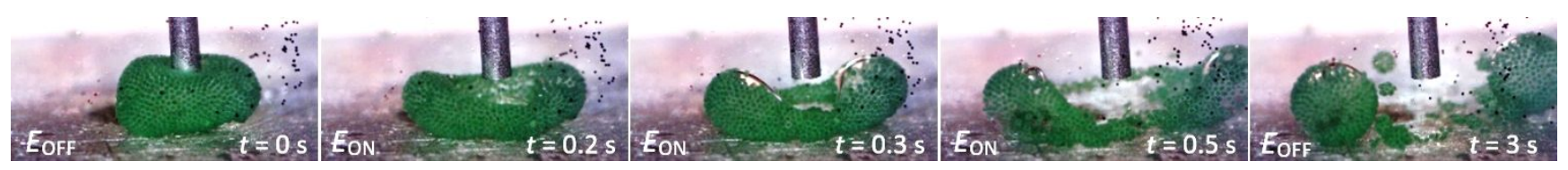

Figure S4. An electrode inserted into the PE particle covered silicone oil droplet ( $2 \mathrm{~mm})$. High electric tension ( $1 \mathrm{kV})$ applied to the electrode caused the droplet to violently break apart.

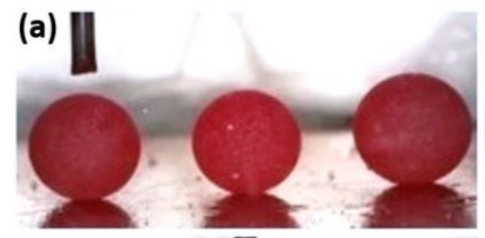

(d)

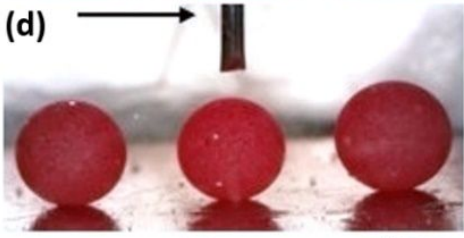

(g)

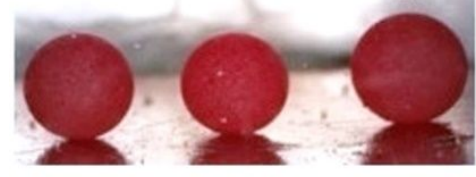

$E=0 \mathrm{~V} \cdot \mathrm{mm}^{-1}$

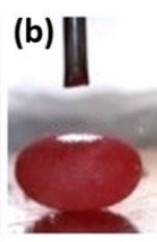

(e)

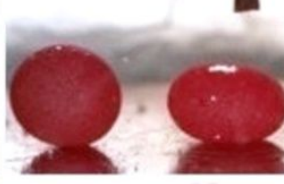

(h) (c)

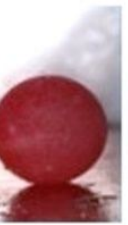

(f)

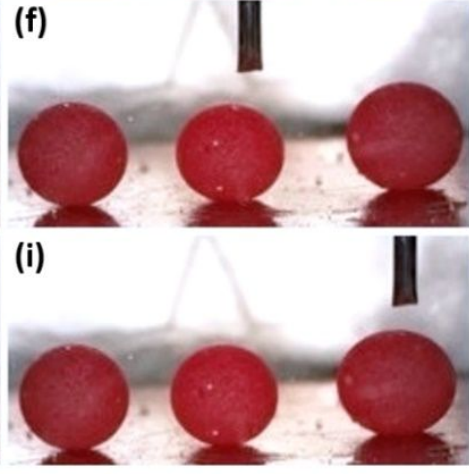

$E=0 \mathrm{~V} \cdot \mathrm{mm}^{-1}$

Figure S5. (a-i) Procedure of inspecting the interior content of particle-covered droplets. Three silicone oil droplets ( $3 \mathrm{~mm})$ were covered with PE particles and placed on a flat electrode in the bottom of the sample cell. A metal rod was used as the second electrode and placed at the center above one of the droplets. Under the application of an electric field (strength 400 $\mathrm{V} \cdot \mathrm{mm}^{-1}$ in vertical direction), the PE particles moved away from the droplet's electric pole and formed an opening in the particle shell that enabled inspection of the inside liquid. See also the corresponding Movie $\mathbf{S 7 .}$

We made three particle-covered droplets $(\sim 3 \mathrm{~mm})$ and placed them on an electrode at the bottom of a sample cell that was filled with castor oil. Above the droplets, we placed a conductive rod-shaped electrode that could move relative to the droplets. Initially, the three droplets were entirely covered with PE particles (Figure S5a). A non-uniform DC electric field of strength $\sim 400 \mathrm{~V} \cdot \mathrm{mm}^{-1}$ was then generated in the vertical direction. The configuration of the electrodes set up a local electric field that only deformed and induced flows around the droplet positioned between the electrodes. Consequently, the PE particles at the interface of that particular droplet moved away from the droplet's electric pole, forming an opening in the particle shell (Figure S5b). Next, we turned off the electric field (Figure S5c) and placed the electrode above a second droplet (Figure S5d). The electric field was then reapplied to form an opening in the PE particle shell (Figure S5e). The same procedure was repeated for the last droplet (Figure $\mathbf{5 g - i}$ ). See also the corresponding Movie S7. 


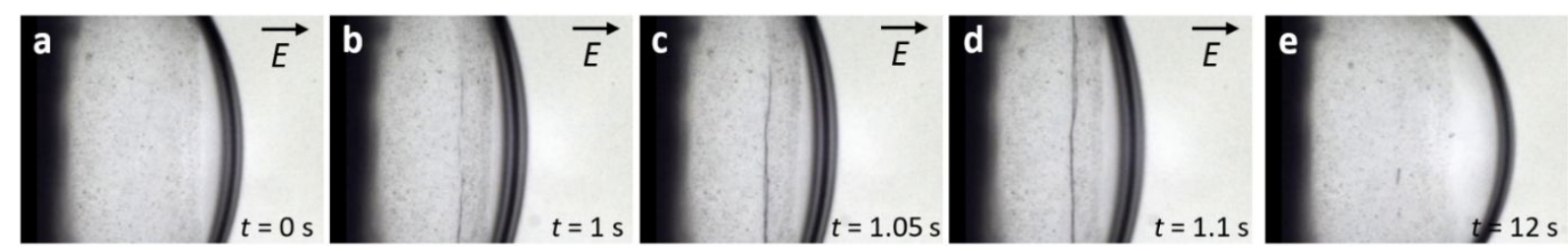

Figure S6. Crumpling of a particle film composed of Laponite clay particles (size $\sim 5 \mu \mathrm{m}$ ) formed on a silicone oil droplet. (a) The particle-covered droplet was subjected to an electric field (horizontal direction) of strength $340 \mathrm{~V} \cdot \mathrm{mm}^{-1}$. (b-d) When the electric field strength was increased to $360 \mathrm{~V} \cdot \mathrm{mm}^{-1}$, the particle film crumpled through one main fold. (e) The particle layer unfolded after the electric field was turned off. See also the corresponding Movie S8. The electric field (through EHD liquid flows) can be used to study mechanical properties of particle films, such as the elasticity and bending stiffness.

Movie S1. Silicone oil droplet covered with polyethylene red particles (REDPMS-0.98, average size $\sim 50 \mu \mathrm{m}$ and density $\sim 0.98$ $\mathrm{g} \cdot \mathrm{cm}^{-1}$ ) were made inside the castor oil using a micropipette. The particles were transported at the silicone oil droplet interface by applying a DC electric field of strength $200 \mathrm{~V} \cdot \mathrm{mm}^{-1}$.

Movie S2. A polyethylene green particle (GPMS-0.98, average size $\sim 20 \mu \mathrm{m}$ and density $\sim 0.98 \mathrm{~g} \cdot \mathrm{cm}^{-1}$ )-covered silicone oil droplet (diameter $\sim 3 \mathrm{~mm}$ ) immersed in castor oil and docked in a nonconductive O-ring (outer diameter of $\sim 4 \mathrm{~mm}$, inner diameter of $\sim 2.5 \mathrm{~mm}$ ) that was fastened to an ITO-coated electrode. The droplet was subjected to a DC electric field. The electric field strength increased stepwise from 0 to $290 \mathrm{~V} \cdot \mathrm{mm}^{-1}$ and then decreased stepwise to $0 \mathrm{~V} \cdot \mathrm{mm}^{-1}$. The movie was sped up 50 times.

Movie S3. A polyethylene red particle (REDPMS-0.98, average size $\sim 50 \mu \mathrm{m}$ and density $\sim 0.98 \mathrm{~g} \cdot \mathrm{cm}^{-1}$ )-covered silicone oil droplet (diameter $\sim 2.5 \mathrm{~mm}$ ) immersed in castor oil and placed on a flat copper electrode at the bottom of a sample cell. A plate electrode was immersed in the castor oil from above. The droplet was subjected to a DC electric field of strength 250 $\mathrm{V} \cdot \mathrm{mm}^{-1}$. The movie was sped up 5 times and looped twice. The closing of the shell in the movie is actually an opening of the shell played in reverse.

Movie S4. A polyethylene green particle (GPMS-0.98, average size $\sim 20 \mu \mathrm{m}$ and density $\sim 0.98 \mathrm{~g} \cdot \mathrm{cm}^{-1}$ )-covered silicone oil droplet (diameter $\sim 3.2 \mathrm{~mm}$ ) immersed in castor oil and docked in a nonconductive O-ring that was fastened to an ITO-coated electrode. The droplet was subjected to a DC electric field of strengths $325-475 \mathrm{~V} \cdot \mathrm{mm}^{-1}$, and the electric field was applied until the particle-free area at drop pole reached the defined value $A_{c 1}(\sim 55,000$ square pixels). The movie was sped up 2 times.

Movie S5. A polyethylene green particle (GPMS-0.98, average size $\sim 90 \mu \mathrm{m}$ and density $\sim 0.98 \mathrm{~g} \cdot \mathrm{cm}^{-1}$ )-covered silicone oil droplet (diameter $\sim 3.5 \mathrm{~mm}$ ) immersed in castor oil and docked in a nonconductive O-ring that was fastened to an ITO-coated electrode. The viscosity of the castor oil was changed by heating the oils from $23{ }^{\circ} \mathrm{C}$ to $50{ }^{\circ} \mathrm{C}$. The silicone oil droplet was subjected to a DC electric field of strength $250 \mathrm{~V} \cdot \mathrm{mm}^{-1}$ until the opening area at the electric pole reached $A_{\mathrm{c} 2}(\sim 45,000$ square pixels). The movie was sped up 2 times.

Movie S6. A polyethylene green particle (GPMS-0.98, average size $\sim 20 \mu \mathrm{m}$ and density $\sim 0.98 \mathrm{~g} \cdot \mathrm{cm}^{-1}$ )-covered silicone oil droplet (diameter $\sim 3.5 \mathrm{~mm}$ ) immersed in castor oil and attached to a nonconductive O-ring (outer diameter of $\sim 4 \mathrm{~mm}$, inner diameter of $\sim 2.5 \mathrm{~mm}$ ) that was fastened to an ITO-coated electrode. The droplet was subjected to a DC electric field of strength $260 \mathrm{~V} \cdot \mathrm{mm}^{-1}$. During the whole experiment, the droplet was illuminated with laser light (wavelength $532 \mathrm{~nm}$, diameter $\sim 0.5 \mathrm{~mm}$, intensity $30 \mathrm{~mW}$ ) at the center of the droplet.

Movie S7. A polyethylene red particle (REDPMS-0.98, average size $\sim 50 \mu \mathrm{m}$ and density $\sim 0.98 \mathrm{~g} \cdot \mathrm{cm}^{-1}$ )-covered silicone oil droplet (diameter $\sim 3 \mathrm{~mm}$ ) immersed in castor oil and placed on a flat copper electrode at the bottom of a sample cell. A metal rod, constituting a second electrode, was placed at the center above the droplets and subjected to a DC electric field of strength $400 \mathrm{~V} \cdot \mathrm{mm}^{-1}$. The movie was sped up 2 times.

Movie S8. A Laponite particle (purchased from Laponite Inc., in the form of a fine white powder)-covered silicone oil droplet (diameter $\sim 5 \mathrm{~mm}$ ) immersed in castor oil and attached to a nonconductive O-ring (outer diameter of $\sim 4 \mathrm{~mm}$, inner diameter of $\sim 2.5 \mathrm{~mm}$ ) that was fastened to an ITO-coated electrode. The droplet was subjected to an electric field of strength 0-360 $\mathrm{V} \cdot \mathrm{mm}^{-1}$ (frequency $0.5 \mathrm{~Hz}$ ). When the electric field strength was increased from 340 to $360 \mathrm{~V} \cdot \mathrm{mm}^{-1}$, the particle film crumpled through one main vertical fold. The movie was sped up 2 times. 